\title{
Distribution of magnetic flux density at the solar surface
}

\section{Formulation and results from simulations}

\begin{abstract}
O. Steiner ${ }^{\star}$
Kiepenheuer-Institut für Sonnenphysik, Schöneckstrasse 6, 79104 Freiburg, Germany

Received 11 March 2003 / Accepted 13 May 2003

Abstract. A formal description of the distribution of magnetic flux density in a quiet Sun region supplemented by an example and an application is presented. We define a flux-based probability density, which is useful to reveal the presence of any strong-field component in the region. The corresponding flux-based probability distribution gives the fraction of the total absolute magnetic flux with a given field-strength limit. Application to the simulations of convective field intensification of Grossmann-Doerth et al. (1998) shows, that, depending on the strength of the initial homogeneous vertical field, 1-50\% of the total magnetic flux within the computational domain of 3 arcsec width is concentrated into flux fibrils with a flux density exceeding $0.1 \mathrm{~T}$. It is shown that a low efficiency of the flux-concentration process is compatible with new observations that suggest a large fraction of the surface magnetic field to have a flux density below $0.1 \mathrm{~T}$.
\end{abstract}

Key words. Sun: magnetic fields - Sun: photosphere - MHD

\section{Introduction}

Results of recent observational and theoretical studies compel to question the conventional picture of the distribution of magnetic flux density at the solar surface of the quiet Sun. The conventional picture holds that more than $90 \%$ of the magnetic flux would occur in concentrated form of flux fragments or flux tubes with a density $>0.1 \mathrm{~T}$, occupying only a few tenths of one percent of the solar surface, while the rest $10 \%$ was to attribute to a weak, turbulent field of less than $0.01 \mathrm{~T}$ strength. For reviews see Stenflo (1989), Stenflo (1994, chapter 12), Solanki (1993, chapter 5.1). The dichotomy of strong flux tubes and a weak, turbulent field has earlier been refuted by the measurements of Livingston (1991) and Rüedi et al. (1992), which revealed flux densities between 0.04 and $0.1 \mathrm{~T}$ in regions with a low magnetic filling factor. These authors used the near infrared line Fe I $1564.85 \mathrm{~nm}$, which allows for a direct measurement of weak fields from the Zeeman splitting. Also Rabin (1992), using the same line, measured true field strengths between 0.075 and $0.1 \mathrm{~T}$. The $90 \%$-limit may still hold, but more likely it becomes subject to revision, too. It is important to remember that the $90 \%$-statement is subject to the condition of a limited spatial resolution of the magnetographic measurement (Frazier \& Stenflo 1972; Howard \& Stenflo 1972) and is therefore of relative value. It cannot be ruled out that a substantial fraction of the total absolute (unsigned) magnetic flux is of mixed polarity on a scale below the spatial resolution of the instrument and thus, escapes polarimetric measurements that are based on the

\footnotetext{
* e-mail: steiner@kis.uni-freiburg.de
}

Zeeman effect. In fact it seems that with ever increasing spatial resolution of polarimetric measurements more and more of medium strong magnetic field is discovered.

Lin (1995) measured the distribution of the magnetic flux density in both, active and quiet regions, using the Michigan State University Infrared Array (MIRC) camera to record Stokes-V profiles of the infrared Fe I lines $\lambda \lambda 1.5648$ and $1.5652 \mu \mathrm{m}$. For the quiet regions he measures a bimodal distribution with a dominant weak field component at around $0.05 \mathrm{~T}$ and a strong field component at approximately $0.14 \mathrm{~T}$. A similar bimodal distribution was found by Lites (2002) from Stokes data recorded with the Advanced Stokes Polarimeter (ASP) comprising the pair of Fe I lines at $630 \mathrm{~nm}$, which limits the analysis to network fields and their immediate surroundings (the network halo). While the core of the magnetic network consists of magnetic elements of $\approx 0.15 \mathrm{~T}$, the second peak in the distribution, near $0.04 \mathrm{~T}$, stems from the halo of polarization surrounding the strong network fields.

Collados (2001) investigates "an extremely quiet Sun region", void of network fields, with the Tenerife Infrared Polarimeter (TIP). He uses the same lines like Lin but different from Lin (1995) and Lites (2002), for this region the probability for finding a given flux density at a given location decreases exponentially with field strength from $0.02 \mathrm{~T}$ onwards, without displaying any clear signal in the range $>0.1 \mathrm{~T}$. This implies that almost none of the flux in the internetwork occurs in strong flux tubes. All these measurements together suggest that the distribution of flux density depends on the region under consideration, which is incompatible with the 
proposition by Stenflo \& Holzreuter (2003a,b) of a universal distribution function for scales of the solar radius down to the dissipation scale. Steiner (2003) suggests that rather, the different distributions might be expression of different physical processes that structure the magnetic field at the solar surface at different scales. Examples include the fast dynamo (Cattaneo 1999) or flux concentration to thermal equipartition values by the convective collapse (Spruit 1979), or by thermal relaxation (van Ballegooijen 1984; Sánchez Almeida 2001). For a discussion on systematic errors in the determination of the field strength derived from visible and infrared lines see Socas-Navarro (2003) and Bellot Rubio \& Collados (2003). It should be mentioned that measurements of the flux density and histograms derived thereof depend on model concepts and inversion techniques. For example, Sánchez Almeida \& Lites (2000) and Socas-Navarro \& Sánchez Almeida (2002) conclude from the same data set as Lites (2002) that "a significant fraction" of the quiet-Sun contains fields exceeding $0.1 \mathrm{~T}$, even for the internetwork, without a bimodal distribution.

Numerical simulations of the solar surface layers comprising the photosphere and the top part of the convection zone result in a distribution of the magnetic flux density that does not show a sharp separation between fields of thermal equipartition energy and weak field either. Cattaneo (1999) obtains an exponential decrease in the probability density of the field strength from weak to strong, where fields of thermal equipartition strength represent just the peaks in the field fluctuation of the fast dynamo driven by the turbulent motion of convection. Stein et al. (2003) obtain from a three-dimensional magnetohydrodynamic simulation including radiation transfer probability densities that show either an exponential decrease or a bimodal structure with a distinct component of $0.15 \mathrm{~T}$, depending on the initial and boundary condition for the magnetic field. In a similar simulation Vögler et al. (2003) and Vögler \& Schüssler (2003) start with a homogeneous, vertical field of a flux density of $0.02 \mathrm{~T}$, which they superimpose on a relaxed convection state, and obtain, after relaxed magnetoconvection, a bimodal probability density, consistent with Stein et al. (2003). The influence of initial and boundary conditions for the magnetic field on the resulting distribution of the flux density is a matter that needs further to be investigated. Here, we ask whether the convective-collapse scenario is in principle compatible with a continuous distribution of field strength with a large fraction of flux in the weak state, or whether it inevitably must lead to a high degree of field concentration, which was thought to be the case for an explanation of the conventional picture.

The above mentioned studies, all show histograms of the distribution of either the absolute value or the vertical or lineof-sight component of the magnetic flux density on the solar surface. In Sect. 2 of this paper we propose a flux-based probability density and distribution, which immediately shows the fraction of the absolute magnetic flux with a given field strength limit, thus useful for testing the conventional $90 \%$-statement. We give examples and apply this formulation to the results of the field intensification simulations of Grossmann-Doerth et al. (1998) in Sect. 3. The results of this application are discussed in Sect. 4 and conclusions are given in Sect. 5.

\section{Probability density function and probability distribution of the magnetic flux density}

The probability that the flux density assumes a value $<B$ at a random location in a certain field of view is given by

$P(B)=\int_{-\infty}^{B} p\left(B^{\prime}\right) \mathrm{d} B^{\prime}$,

where $P(B)$ is called the probability distribution, $p(B)$ is the probability density function (PDF), or for short, probability density, and $B$ may assume values in the interval $-\infty<B<+\infty \quad$ (Sachs 1999; Zelen \& Severo 1970). Considering a finite field of view of area $A$, the probability for the field strength at a given location within this area, $\hat{B}$, to have a value $<B$ is equal to the area that harbours a flux density with this property divided by the total area of the field of view from where it follows that

$p(B)=\frac{1}{A} \int_{A} \delta(B-\hat{B}) \mathrm{d} a$.

We can think of $B$ as, e.g., the vertical component of the flux density vector at the solar surface, $B_{z}$, or the line-of-sight component, $B_{\mathrm{LOS}}$, or the absolute value of $\boldsymbol{B},|\boldsymbol{B}|$, or $\operatorname{sign}\left(B_{z}\right)|\boldsymbol{B}|$.

Next we imagine our field of view to be subdivided into infinitesimal flux elements with equal amounts of magnetic flux, $|\mathrm{d} \Phi|$. Then, the probability that a given flux element, $|\mathrm{d} \Phi|$, has a flux density with a value in the interval $\left[B_{0}, B_{0}+B\right]$ is equal to the absolute magnetic flux trough the area $A$ that has this same property, divided by the total amount of absolute flux, $|\Phi|_{\text {tot }}$. Correspondingly, we can define a "flux-based" probability density given by

$p_{\Phi}(B)=\frac{1}{|\Phi|_{\text {tot }}} \int_{A} \delta(B-\hat{B})\left|\hat{B}_{z}\right| \mathrm{d} a$.

For the two special cases of $B=B_{z}$ and $B=\left|B_{z}\right|$ it follows from Eqs. (2) and (3),

$p_{\Phi}\left(B_{z}\right)=\frac{\left|B_{z}\right| p\left(B_{z}\right)}{\int_{-\infty}^{+\infty}\left|B_{z}\right| p\left(B_{z}\right) \mathrm{d} B_{z}}, \quad$ and
$p_{\Phi}\left(\left|B_{z}\right|\right)=\frac{\left|B_{z}\right| p\left(\left|B_{z}\right|\right)}{\int_{0}^{+\infty}\left|B_{z}\right| p\left(\left|B_{z}\right|\right) \mathrm{d}\left|B_{z}\right|}$.

As illustrative examples, we consider an exponential and a Gaussian probability density representing two different magnetic components, and the combination of the two for a region consisting of these two components. The exponential probability density is given by

$p_{\mathrm{e}}(x)=\alpha \mathrm{e}^{-\alpha x} \quad x \geq 0$.

The random variable $x$ can be thought to stand for $\left|B_{z}\right|$. From Eq. (5) we then obtain

$p_{\mathrm{e} \Phi}(x)=\alpha^{2} x \mathrm{e}^{-\alpha x}=\alpha x p_{\mathrm{e}}(x)$.

The Gaussian probability density is given by

$p_{\mathrm{G}}(x)=\frac{1}{\sqrt{2 \pi} \sigma} \mathrm{e}^{-\frac{1}{2} \frac{(x-a)^{2}}{\sigma^{2}}}$. 

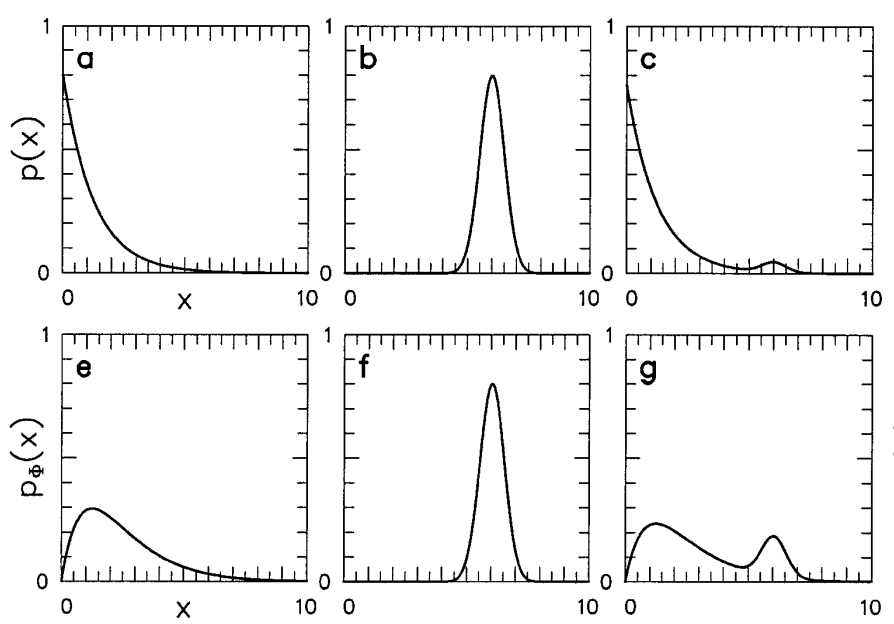

From Eq. (5) and under the condition $a \gg \sigma$, we obtain

$p_{\mathrm{G} \Phi}(x) \approx \frac{1}{\sqrt{2 \pi} \sigma a} x \mathrm{e}^{-\frac{1}{2} \frac{(x-a)^{2}}{\sigma^{2}}}=\frac{x}{a} p_{\mathrm{G}}(x)$.

For the combination of the two we use a fill factor $f \leq 1$ so that

$p_{\mathrm{c}}(x)=f p_{\mathrm{e}}(x)+(1-f) p_{\mathrm{G}}(x)$

and

$p_{\mathrm{c} \Phi}(x)=\frac{f p_{\mathrm{e} \Phi}(x)+(1-f) a \alpha p_{\mathrm{G} \Phi}(x)}{f+(1-f) a \alpha}$.

The probability densities of Eqs. (6)-(11) including the probability distribution for the combined cases are shown in Fig. 1, where the following parameters have been chosen: $\alpha=0.8$, $\sigma=0.5, a=6.0$, and $f=0.95$. The top row shows from left to right the exponential and Gaussian probability densities according to Eqs. (6) and (8), the combined probability density of Eq. (10), and the probability distribution obtained by numerical integration of Eq. (10). From panel $\mathrm{c}$ we see that with a fill factor of $5 \%$, the strong-field component is barely discernible in the probability density or probability distribution and might easily go unnoticed in a superficial analysis. Remedy is provided by the flux-based probability density, $p_{\Phi}$, which more clearly displays the strong field component as can be seen in panel g. Another advantage of this method consists in providing the fraction of the total magnetic flux with a given property, which allows for a direct test of the $90 \%$-statement, recalled in Sect. 1. For example, from panel $\mathrm{h}$ we find that $28 \%$ of the total flux has a strength $>5$.

Given the probability density $p_{x}(x)$, the probability density $p_{u}(u)$ for the new random variable $u$, with the strictly monotonic transformation, $x=f(u)$, is given by

$p_{u}(u)=\frac{\mathrm{d} P_{u}(u)}{\mathrm{d} u}=p_{x}(x) \cdot \frac{\mathrm{d} x}{\mathrm{~d} u}=p_{x}(f(u)) \frac{\mathrm{d} f(u)}{\mathrm{d} u}$.

We can use this relation for deriving probability densities for the "field energy" in which case $u \propto B^{2}$. Applying this transformation to our three exemplary probability densities reveals that they become singular at $u=0$, but this singularity disappears when using the flux-based probability density.

In practice we do not have a continuous probability density available, but instead, given the finite number of pixels or grid
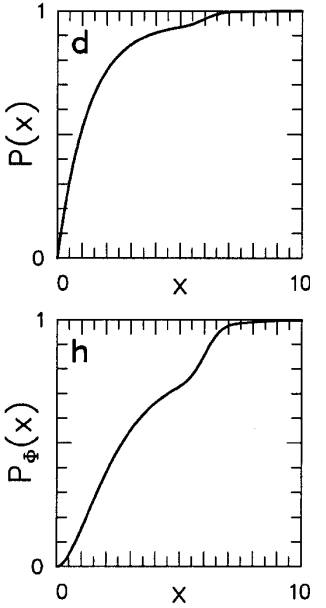

Fig. 1. Continuous probability densities and associated probability distributions. a) Exponential and b) Gaussian probability density and c) a linear combination of a) and b) with weight factors $f$ and $1-f$, respectively, where $f=0.95$. d) Probability distribution for the combined case c). e)-g) Flux-based probability densities corresponding to a)-c), respectively. h) Flux-based probability distribution of the combined case $\mathbf{g}) . P_{\Phi}(x)$ renders the fraction of the total flux that has a field strength $<x$. E.g., $72 \%$ of the total flux has a strength less than 5 .

cells from observations or numerical simulations, respectively, we establish a histogram of the measured quantity in order to obtain a qualitative view of the probability density.

Subdividing the range of values of $N_{\text {pix }}$ measurements, $B_{i}$, $i=1 \ldots N_{\text {pix }}$, into $N_{\text {class }}$ class-intervals, the discrete probability density for the $k$ th interval, $\left[B_{k}, B_{k}+\Delta B\right.$ ], is given by

$p_{k}=\frac{1}{N_{\text {pix }} \Delta B} \sum_{\substack{B_{k} \leq B_{i}<B_{k}+\Delta B \\ i=1}}^{N_{\text {pix }}} 1$,

where the sum on the r.h.s. of Eq. (13) is the absolute frequency and $\Delta B$ the (constant) class interval. For the flux-based probability density we correspondingly obtain

$p_{\Phi k}=\frac{1}{\Delta B|\Phi|_{\text {tot }}} \sum_{\substack{B_{k} \leq B_{i}<B_{k}+\Delta B \\ i=1}}^{N_{\mathrm{pix}}}\left|B_{z i}\right| A_{i}$,

where $A_{i}$ is the area of the $i$ th pixel or grid cell. With $A_{i}=$ $A / N_{\text {pix }}=$ const and $\left\langle\left|B_{z}\right|\right\rangle_{k}$ the arithmetic mean of the vertical components of all pixels belonging to class $k$ we obtain the following correspondence between $p_{k}$ and $p_{\Phi k}$ :

$p_{\Phi k}=\frac{1}{\Delta B|\Phi|_{\text {tot }}} \frac{A}{N_{\text {pix }}}\left\langle\left|B_{z}\right|\right\rangle_{k} \sum_{\substack{B_{k} \leq B_{i}<B_{k}+\Delta B \\ i=1}}^{N_{\text {pix }}} 1=\frac{\left\langle\left|B_{z}\right|\right\rangle_{k} p_{k}}{\Delta B \sum_{k=1}^{N_{\text {class }}}\left\langle\left|B_{z}\right|\right\rangle_{k} p_{k}}$

\section{Results from MHD-simulations}

The structuring of magnetic flux at the solar surface presumably originates from different physical processes as pointed out in Sect. 1. One candidate process is the convective collapse, a plasma instability that ensues if a magnetic flux tube is embedded in a superadiabatically stratified medium (Parker 1978; Spruit 1979). It leads either to a concentration of the flux density up to the thermal equipartition value or to a flux dispersal, depending on whether the perturbation of the initial equilibrium configuration is in the upward or the downward direction. The instability draws its energy from the superadiabaticity of the top layer of the convection zone. In this sense it is not different from the regular thermal convective instability, except that the presence of a strong enough magnetic field does prevent it from onset (Spruit \& Zweibel 1979). 

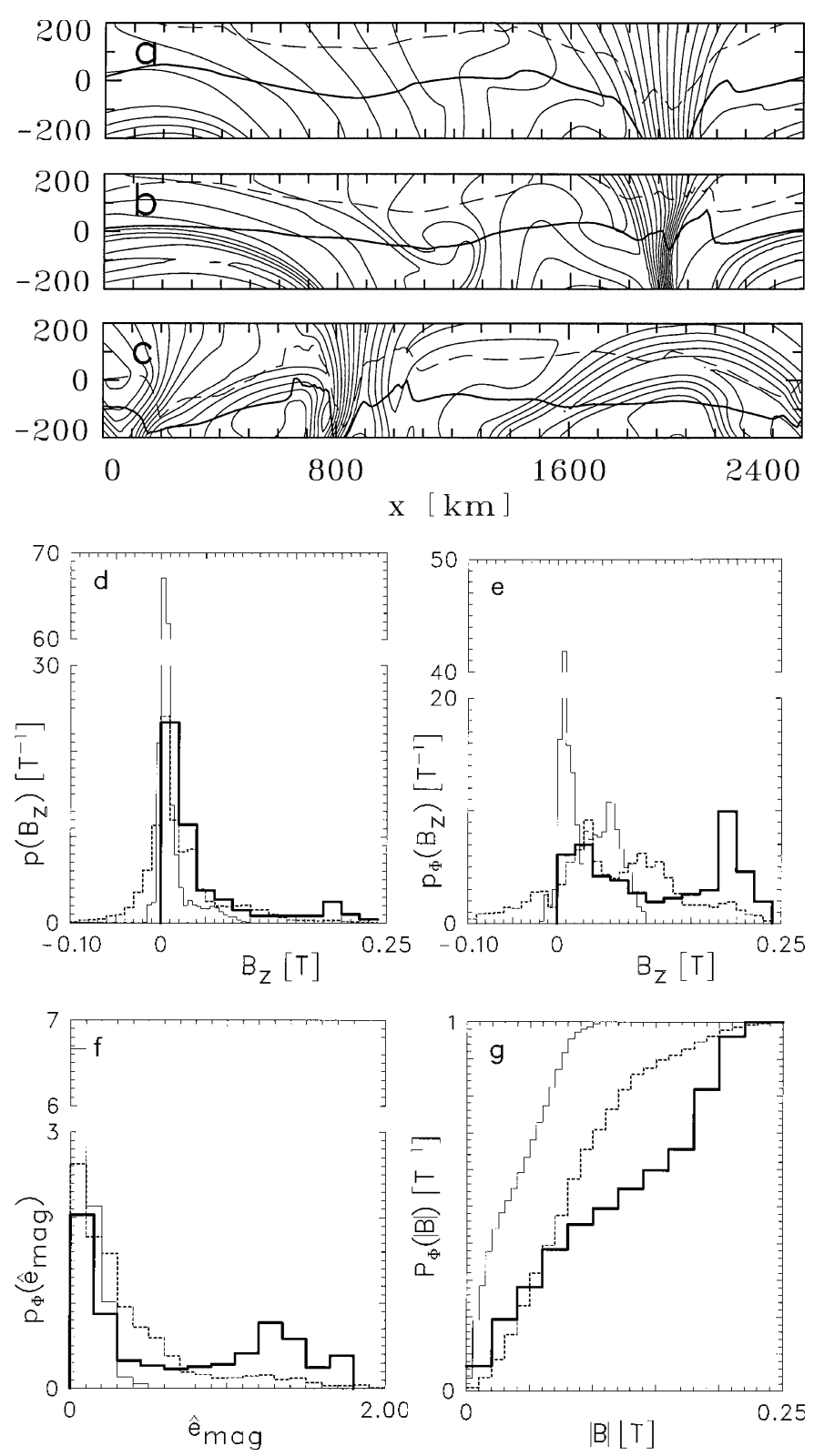

Fig. 2. a)-c) Three time instants of magnetic lines of force shortly after a convective collapse has taken place, which led to the flux concentrations at $x \simeq 1900 \mathrm{~km}$ in panels a) and b) and at $x \simeq 200 \mathrm{~km}$ and $x \simeq 900 \mathrm{~km}$ in panel c). The horizontally running curves indicate surfaces of optical depth $\tau_{500 \mathrm{~nm}}=1$ (solid) and $\tau_{500 \mathrm{~nm}}=0.1$ (dashed). $z$ is the coordinate in the vertical direction. d)-g) Discrete probability densities and distributions of the magnetic field density along the surface of optical depth $\tau_{500 \mathrm{~nm}}=0.1$ of panels a)-c), where the functions plotted with heavy lines correspond to panel a), thin lines to panel b) and dotted lines to panel c). d) Histogram (probability density function) of the vertical magnetic flux density, $B_{z}$. e) Flux-based probability density $B_{z}$. f) Probability density for the magnetic energy $e_{\text {mag }}=|\boldsymbol{B}|^{2} /(8 \pi)$, normalized to the energy of a fixed thermal equipartition field strength of 0.17 T. g) Flux-based probability distribution indicating the fraction of the total absolute flux that has an absolute flux density of less than $|B|$.

A two-dimensional magnetohydrodynamic simulation of field intensification by the convective collapse instability taking compressibility and radiative transfer into account has been carried out by Steiner (1996) and Grossmann-Doerth et al. (1998). An unexpected result of these simulations is that, depending on the magnetic flux taking part in the process, a "rebound shock" can form as a consequence of the strong downflow within the collapsing tube, leading to a subsequent upflow and flux dispersal. Here, we do not further go into the details of these simulations (see also Steiner 1999) but concentrate on the probability density of the resulting surface magnetic field. Note that these results refer to single convective-collapse events and their close vicinity and not to a relaxed magnetoconvective state.

The simulations start with a homogeneous vertical magnetic field of a given field strength, $B_{z \text { init }}$, where we investigate the three cases with $B_{z \text { init }}=0.01,0.02$, and $0.04 \mathrm{~T}$, corresponding to the simulation runs $\mathrm{C} 1, \mathrm{C} 2$, and $\mathrm{C} 4$ of Grossmann-Doerth et al. (1998), and to panels b, c, and a of Fig. 2, respectively. Figures $2 \mathrm{a}-\mathrm{c}$ show for each case magnetic lines of force in a narrow height range of $400 \mathrm{~km}$ around the $\tau_{500 \mathrm{~nm}}=1$ surface at a time instant shortly after the convective collapse has taken place and, $150 \mathrm{~s}, 200 \mathrm{~s}$, and $250 \mathrm{~s}$, respectively, after the initial state of a homogeneous vertical magnetic field.

The probability density of the initial condition can be expressed in terms of a $\delta$-distribution: $p\left(B_{z}\right)=\delta\left(B_{z \text { init }}-B_{z}\right)$. One would expect that, after the convective collapse has taken place, the " $\delta$-peak" were shifted to the thermal equipartition value of $B \approx 0.15 \mathrm{~T}$ and broadened to some extent because of diffusion effects taking place. Instead, Fig. $2 d$ shows discrete probability densities for $B_{z}$ with an exponential-like core and only a modest or no peak in the range of the thermal equipartition field-strength, although clearly flux tubes have formed as can be seen from the field-line plots of panels a-c.

Here and in the following, the magnetic field is measured along a curve that delineates optical depth $\tau_{500 \mathrm{~nm}}=0.1$, which curve runs approximately parallel to and $140 \mathrm{~km}$ above the curve of optical depth $\tau_{500 \mathrm{~nm}}=1.0$, also indicated in Figs. 2a-c. In panels d-g curves plotted with heavy lines, correspond to the snapshot of panel a $\left(B_{z \text { init }}=0.04 \mathrm{~T}\right)$, thin-line curves to the case of panel $\mathrm{b}\left(B_{z \text { init }}=0.01 \mathrm{~T}\right)$, and the dotted curves to panel c $\left(B_{z \text { init }}=0.02 \mathrm{~T}\right)$. In order to improve the statistics, the histograms of the the latter two cases are based on data as a function of space and time. Time runs from 60 to $230 \mathrm{~s}$, in case of the thin-line histograms, and from 100 to $300 \mathrm{~s}$ in case of the dotted-line histograms. Both time periods are about half a granular life-time.

We first concentrate on the solid-line histograms of Fig. 2 and note that the modest peak in $p\left(B_{z}\right)$ between 0.18 and $0.22 \mathrm{~T}$, which stems from the flux tube that has formed at around $x=1900 \mathrm{~km}$, becomes the dominant peak in the corresponding PDF of panel e, which shows the corresponding flux-based probability density, $p_{\Phi}\left(B_{z}\right)$, introduced with Eqs. (3) and (14). The discrete probability distributions, $P_{\Phi}\left(B_{z}\right)$, shown in Fig. $2 \mathrm{~g}$ (which are integrals of PDFs similar to the ones shown in panel e but for the absolute field strength, $|\boldsymbol{B}|$ ) indicate the fraction of the total magnetic flux through the $\tau_{500 \mathrm{~nm}}=0.1$-surface that has a flux density $<B$. From this plot we see that a large fraction of the magnetic flux occurs in the form of weak field despite of the limited width of $2400 \mathrm{~km} \approx 3 \operatorname{arcsec}$ of the 
computational domain within which the convective collapse has taken place. Inspecting panel a we readily see that not all of the field has taken part in the convective collapse. Weak field is left over atop the granular upwelling, where flux dispersal takes place, so that in this case still $50 \%$ of the total absolute flux appears in the form of field with a strength of less than $0.1 \mathrm{~T}$, as can be read off the heavy-line curve of panel $\mathrm{g}$.

Figure $2 \mathrm{f}$ shows the flux-based probability densities expressed in terms of the magnetic energy, $\hat{e}_{\text {mag }} \propto B^{2}$, normalized to the thermal equipartition energy at the solar surface corresponding to a field of $0.17 \mathrm{~T}$. Since these histograms show classes of equidistant energy increments, the strong field component of the heavy-line curve is spread out over many classes, while all the flux densities below $0.1 \mathrm{~T}$ fall into the first four classes.

The time series $\mathrm{C} 1$, represented by the instant shown in panel $b$, starts from the same flow pattern as does run $\mathrm{C} 4$, except that the initial field strength is $B_{z \text { init }}=0.01 \mathrm{~T}$. Therefore, panels $\mathrm{a}$ and $\mathrm{b}$ resemble each other but due to the smaller amount of magnetic flux the width of the flux sheet in the case of Fig. $2 b$ is much smaller. This leads to a less vigorous convective collapse, because the thin magnetic flux sheet is efficiently heated by lateral influx of radiation, which has a decelerating effect on the downflowing gas. As a consequence, the field gets less amplified and the flux sheet, although clearly visible in panel $b$, is hardly discernible from the histogram of the vertical component of the magnetic flux density, shown in panel d (thin line). It becomes not visible until plotting the flux-based probability density, shown in panel e, where a peak is seen between 0.04 and $0.09 \mathrm{~T}$. From the probability distribution shown in panel $\mathrm{g}$ we see that more than $98 \%$ of the total absolute flux has a field strength at $\tau_{500 \mathrm{~nm}}=0.1$ of less than $0.1 \mathrm{~T}$.

Figure $2 \mathrm{c}$ is a snapshot from the time series $\mathrm{C} 2$, which starts from a different flow pattern than do run $\mathrm{C} 4$ and $\mathrm{C} 1$ and which has an initial field strength of $B_{z \text { init }}=0.02 \mathrm{~T}$. In this case two flux concentrations form at $x \simeq 100 \mathrm{~km}$ and $x \simeq 800 \mathrm{~km}$, where the latter is more deeply rooted and stronger. In this case a more symmetric probability density for $B_{z}$ evolves (dotted line, panel d). Since two field concentrations of different strengths and since additional, horizontal field concentrations above the convective upwelling are present, weak and strong field are less clearly separated as can be seen from the probability densities of panels d-f. A peak around $0.19 \mathrm{~T}$ and $\hat{e}_{\mathrm{mag}} \simeq 1.25$ (corresponding to $0.19 \mathrm{~T}$ ) is visible in panels e and $\mathrm{f}$, respectively. It stems from the flux concentration at $x \simeq 800$. In this case, the flux-based probability distribution tells us that $70 \%$ of the total absolute flux has a field strength of less than $0.1 \mathrm{~T}$.

In all three cases, the weak field component is not restricted to below $0.01 \mathrm{~T}$ but it shows rather an exponentially decreasing probability density from very weak flux density to the strongfield component, leaving ample flux of medium field strength. The distribution of the flux density depends on the initial field strength, $B_{z \text { init }}$, since it influences the course of the convective collapse. For example, if $B_{z \text { init }}=0.01 \mathrm{~T}$, the spatial extent of the flux concentration is so small that the convective collapse is hampered by radiative heating so that no strong-field component with $B>0.1 \mathrm{~T}$ can form. If $B_{z \text { init }}=0.04 \mathrm{~T}, 50 \%$ of the total flux gathers in a strong field component but it gets subsequently dispersed by an upflow developing in connection with the "rebound shock".

While the influence of the initial condition is strong, the boundary conditions are less determining for the interior solution of the magnetic field because the convective collapse takes place within a short period of time compared to the granular time-scale. The values for $P_{\Phi}(B=0.1 \mathrm{~T})$ derived here must be considered as on the low side because of the special configuration of the initial field, which favours onset and development of the convective collapse instability and because of the restriction to one horizontal spatial dimensions, when the flux farthest away from the collapse location that escapes concentration is less than would be in two dimensions.

\section{Discussion}

In the previous section we have analysed single events of convective flux concentrations starting from a homogeneous, vertical field. Even in this ideal situation, the convective collapse concentrates only a fraction of the available total absolute magnetic flux over an area of a granular cell and does not produce flux-tubes of thermal equipartition energy if the initial flux density is $\$ 0.01 \mathrm{~T}$, which corresponds to approximately $10^{10} \mathrm{~W}$. Rajaguru \& Hasan (2000) found from an analysis of radiative transfer effects on the onset of convective collapse a critical flux limit of $\approx 1 \times 10^{10} \mathrm{~W}$, which is compatible with the above rough limit for the case of $B_{z \text { init }}=0.01 \mathrm{~T}$, where amplification to thermal equipartition fails. This limit would explain why possibly no strong flux-tubes are formed by the convective collapse instability in the internetwork region. In regions where it can take place, e.g., the network and plages, the connection of our results of the probability distribution to the observed partition of the magnetic flux into the strong and the weak state involves the creational rate and lifetime of flux tubes and shall be demonstrated by the following idealized model.

Consider an area, $A$, harbouring two components of vertical homogeneous magnetic field of unique polarity, which cover the area completely and together account for the total magnetic flux $\Phi$. The strong-field component consists of $N_{\mathrm{ft}}$ flux tubes of flux $\Phi_{\mathrm{ft}}$ so that the total flux in the strong state is $\Phi_{\mathrm{s}}=N_{\mathrm{ft}} \Phi_{\mathrm{ft}}$. Flux tubes are created with a rate $N_{\mathrm{c}}$ and have a decay time $\tau_{\mathrm{ft}}$. $N_{\mathrm{c}}$ and $\tau_{\mathrm{ft}}$ are constants as they depend on the convective motion, which is thought to evolve independent on the flux partition. Then, the change in strong flux is

$$
\mathrm{d} \Phi_{\mathrm{s}}=N_{\mathrm{c}} \Phi_{\mathrm{ft}} \mathrm{d} t-\frac{N_{\mathrm{ft}}(t)}{\tau_{\mathrm{ft}}} \Phi_{\mathrm{ft}} \mathrm{d} t,
$$

which must vanish after some time when a stationary state has evolved. The flux concentrated in a single tube, $\Phi_{\mathrm{ft}}$, depends on the flux density of the weak component. The creation of a flux tube proceeds by gathering weak flux in a region $A_{\mathrm{g}}$ with an efficiency $\epsilon_{\mathrm{c}} . \epsilon_{\mathrm{c}}$ can be identified with the fraction of the available flux over a granular area that is concentrated to strong field in a collapse event, which relates to our simulation results: $\epsilon_{\mathrm{c}}=1-P_{\Phi}\left(B_{z}=0.1\right)$. Consequently, $\Phi_{\mathrm{ft}}=\epsilon_{\mathrm{c}} A_{\mathrm{g}}\left(\Phi-\Phi_{\mathrm{s}}\right) / A$. If we denote the creational period per gathering area, $A_{\mathrm{g}}$, with $\tau_{\mathrm{g}}$ (which might be about a granular lifetime) and the fraction of 
the total flux in the strong state by $f=\Phi_{\mathrm{s}} / \Phi$ (which can be observed), we obtain in the stationary phase

$f=\frac{\epsilon_{\mathrm{c}}}{\frac{\tau_{\mathrm{g}}}{\tau_{\mathrm{ft}}}+\epsilon_{\mathrm{c}}}$.

If the lifetime of the flux tubes is long, $f \simeq 1$, so that most of the flux is in the strong state irrespective of the efficiency $\epsilon_{\mathrm{c}}$. However, according to Berger \& Title (1996), the time scale of the magnetic flux evolution in active region plages is on the order of the granular lifetime suggesting to set $\tau_{\mathrm{ft}} \simeq \tau_{\mathrm{g}}$ in which case $f \simeq \epsilon_{\mathrm{c}}$ for small values of $\epsilon_{\mathrm{c}}$. But even when $\tau_{\mathrm{ft}}=10 \cdot \tau_{\mathrm{g}}$, $f<0.7$ if $\epsilon_{\mathrm{c}} \leq 0.2$. Then, $\epsilon_{\mathrm{c}}$ cannot be too large if considerably more than $10 \%$ of the total flux ought to be in the weak-field state, which is compatible with the results of Sect. 3, recalling that the values for $P_{\Phi}\left(B_{z}=0.1\right)$ must considered to be rather lower limits.

The reason for the relative inefficiency of the convective collapse in the simulations of Sect. 3 originates in the largely different time scales for the convective collapse of below $1 \mathrm{~min}$ and for the flux gathering process of the order of the turnover time of convection. Only if a sufficient amount of magnetic flux can be transported to the location where a downflow sets in, intensification to thermal equipartition field strength evolves. If this transport is too slow the collapse becomes hampered by radiative damping.

\section{Conclusions}

The following conclusions can be drawn:

1. The flux-based probability density is a useful means in order to reveal any strong-field component that otherwise may go unnoticed when plotting the usual, area-based PDF.

2. The flux-based probability distribution, $P_{\Phi}(B)$ or $P_{\Phi}\left(e_{\text {mag }}\right)$, gives the fraction of the total absolute magnetic flux of maximum field strength, $B$, or magnetic energy, $e_{\text {mag }}$, respectively.

3. Given the data of a field property, e.g, for $|B|, B_{\mathrm{LOS}}$, or $e_{\mathrm{mag}}$, and given the vertical field component, $B_{z}$, it is always possible to convert between the area-based and the flux-based probability density and distribution.

4. Two-dimensional simulations of convective field intensification show that even under favourable conditions, a single convective collapse event concentrates only 1-50\% (depending on the strength of the initial field) of the total available magnetic flux within the computational domain of 3 arcsec into flux fibrils with a flux density exceeding $0.1 \mathrm{~T}$.

5. The existence of a large flux fraction of weak field is not in contradiction with the existence of the convective collapse instability.

Acknowledgements. I thank M. Stix for detailed comments on a draft of this paper and the referee for his proficient critique.

\section{References}

Bellot Rubio, L., \& Collados, M. 2003, A\&A, 406, 357

Berger, T. E., \& Title, A. M. 1996, ApJ, 463, 365

Cattaneo, F. 1999, ApJ, 515, L39

Collados, M. 2001, in Advanced Solar Polarimetry - Theory, Observation, and Instrumentation, ed. M. Sigwarth, ASP Conf. Ser., 236, 255

Frazier, E. N., \& Stenflo, J. O. 1972, Sol. Phys., 27, 330

Grossmann-Doerth, U., Schüssler, M., \& Steiner, O. 1998, A\&A, 337, 928

Howard, R., \& Stenflo, J. O. 1972, Sol. Phys., 22, 402

Lin, H. 1995, ApJ, 446, 421

Lites, B. W. 2002, ApJ, 573, 431

Livingston, W. 1991, in Solar Polarimetry, NSO/SP Workshop No. 11, ed. L. November, 356

Parker, E. N. 1978, ApJ, 221, 368

Rabin, D. 1992, ApJ, 390, L103

Rajaguru, S. P., \& Hasan, S. S. 2000, ApJ, 544, 522

Rüedi, I., Solanki, S. K., Livingston, W., \& Stenflo, J. O. 1992, A\&A, 263, 323

Sánchez Almeida, J. 2001, ApJ, 556, 928

Sánchez Almeida, J., \& Lites, B. W. 2000, ApJ, 532, 1215

Sachs, L. 1999, Angewandte Statistik (Springer)

Socas-Navarro, H. 2003, in Third International Workshop on Solar Polarization, ed. J. Trujillo Bueno, \& J. Sánchez Almeida, ASP Conf. Ser., in press

Socas-Navarro, H., \& Sánchez Almeida, J. 2002, ApJ, 565, 1323

Solanki, S. K. 1993, Space Sci. Rev., 63, 1

Spruit, H. C. 1979, Sol. Phys., 61, 363

Spruit, H. C., \& Zweibel, E. G. 1979, Sol. Phys., 62, 15

Stein, R. F., Bercik, D., \& Nordlund, ^̊. 2003, Il Nuovo Cimento C, 25,513

Steiner, O. 1996, in Solar and Galactic Magnetic Fields, ed. D. Schmitt, \& H. Voigt (Göttingen: Vandenhoeck \& Ruprecht)

Steiner, O. 1999, in Third Advances in Solar Physics Euroconference, ed. B. Schmieder, A. Hofmann, \& J. Staude, ASP Conf. Ser., 184, 38

Steiner, O. 2003, in NATO Advanced Research Workshop Turbulence, Waves, and Instabilities in the Solar Plasma, ed. R. Erdélyi, \& K. Petrovay (Kluwer), in press

Stenflo, J., \& Holzreuter, R. 2003a, in Current Theoretical Models and Future High Resolution Solar Observations, ed. A. Pevtsov, \& H. Uitenbroek, ASP, Conf. Ser., 286, 169

Stenflo, J., \& Holzreuter, R. 2003b, in Magnetic Coupling of the Solar Atmosphere, ed. H. Sawaya-Lacoste (ESA SP-505), IAU Colloq., 188,101

Stenflo, J. O. 1989, A\&ARv, 1, 3

Stenflo, J. O. 1994, Solar magnetic fields: Polarized radiation diagnostics (Kluwer Academic Publishers)

van Ballegooijen, A. 1984, in Small-Scale Dynamical Processes in Quiet Stellar Atmospheres, ed. S. Keil (NSO/SP Workshop Ser. 5, Sunspot, NM), 260

Vögler, A., \& Schüssler, M. 2003, Astron. Nachrichten, in press

Vögler, A., Shelyag, S., Schüssler, M., et al. 2003, in Modelling of Stellar Atmospheres, IAU Symp. 210, ed. N. Piskunov, W. W. Weiss, \& D. F. Gray, ASP Conf. Ser., in press

Zelen, M., \& Severo, N. C. 1970, in Handbook of Mathematical Functions, ed. M. Abramowitx, \& I. Stegun (Dover), 925 